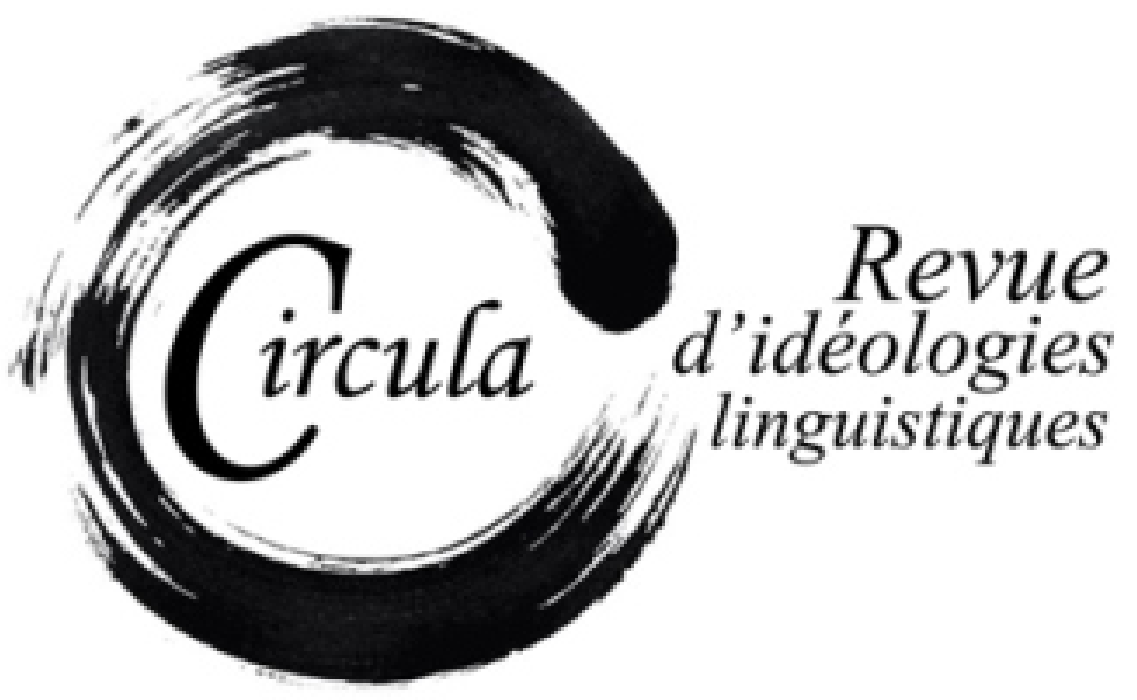

TITRE: LA POLÉMIQUE AUTOUR DE LA NOUVELLE POLITIQUE DE L'EMPRUNT LINGUISTIQUE DE L'OFFICE QUÉBÉCOIS DE LA LANGUE FRANÇAISE

Auteures: Geneviève Bernard Barbeau, Université du QuéBEC À Trols-Rivières et Véronique DUROCHER, UNIVERSITÉ DU QUÉBEC À TROIS-RIVIÈRES

Revue: Circula, numéro 9 : A-t-on encore Peur des anglicismes? PerCePtion actuelle des anglicismes au QuébeC ET DANS L'ESPACE FRANCOPHONE

PAgES: $58-76$

ISSN: 2369-6761

DiRECTRICES: MIREILLE ELCHACAR ET NADINE VINCENT

URI: HTTP://HDL.HANDLE.NET/11143/16047

DOI: HTTPS://DOI.ORG/10.17118/11143/16047 


\title{
La polémique autour de la nouvelle politique de l'emprunt linguistique de l'Office québécois de la langue française
}

\author{
Geneviève Bernard Barbeau, Université du Québec à Trois-Rivières \\ genevieve.bernardbarbeau@uqtr.ca
}

Véronique Durocher, Université du Québec à Trois-Rivières

veronique.durocher@uqtr.ca

Résumé : En janvier 2017, l'Office québécois de la langue française (OQLF) a adopté une nouvelle politique dans laquelle étaient révisés les principes et les critères de traitement sur lesquels se fonde son intervention linguistique en matière d'emprunts linguistiques, et plus particulièrement d'emprunts à l'anglais. Or, la question de l'emprunt à l'anglais est susceptible de susciter nombre de débats et d'échanges polémiques au Québec, et c'est exactement ce qui s'est produit quand l'OQLFa rendu publique sa nouvelle politique. Cet article est consacré aux débats suscités par cette nouvelle politique de l'emprunt dans l'espace médiatique. L'objectif est de montrer la dichotomie qui existe entre les prises de position constitutives du débat et d'ainsi faire état de la perception actuelle non seulement des emprunts à l'anglais, mais également du rapport entre français et anglais au Québec et, plus largement, du rôle de l'OQLF dans la société québécoise.

Mots-clés : anglicismes; Office québécois de la langue française ; polémique ; médias ; Québec

Abstract: In January 2017, the Office québécois de la langue française (OQLF) adopted a new policy revising the principles and the treatment criteria on which its language intervention regarding linguistic borrowing, and more specifically anglicisms, is based. However, the issue of anglicisms is likely to prompt debates in Quebec, and this is exactly what happened when the OQLF published its new policy. This article focuses on the polemic caused by this policy in the media. The aim is to show the dichotomy between the various points of view that have been taken and thus to reflect on the current perception of not only anglicisms, but also the relationship between French and English in Quebec and, more broadly, the role of the OQLF in the society.

Keywords: anglicism; Office québécois de la langue française; polemic; media; Québec 


\section{Introduction}

En janvier 2017, l'Office québécois de la langue française (OQLF) a adopté une nouvelle politique dans laquelle étaient révisés « les principes et les critères de traitement sur lesquels se fonde son intervention linguistique » (OQLF, 2017 : 3) en matière d'emprunts linguistiques, et plus particulièrement d'emprunts à l'anglais. Or, qui connaît la situation sociolinguistique québécoise sait à quel point la question de l'emprunt à l'anglais est délicate et qu'elle est susceptible de provoquer des débats virulents (Bouchard, 1999 ; Vincent, 2017). II n'est alors pas étonnant que la nouvelle politique de l'OQLF ait suscité de tels débats lorsqu'elle a été rendue publique et diffusée dans les médias en septembre 2017, plus de huit mois après son adoption. Ce sont ces débats, articulés autour d'une série d'arguments en faveur et en défaveur de la nouvelle politique, certes, mais aussi plus largement à propos de la question des emprunts à l'anglais au Québec, de la situation sociolinguistique des Québécois et du rôle de l'OQLF dans la société, qui nous intéressent ici.

\section{L'OQLF et sa nouvelle politique de l'emprunt linguistique}

Créé en 1961, l'Office québécois de la langue française (alors Office de la langue française) a initialement comme objectif de « veiller, sous le mandat du ministre [alors aux Affaires culturelles] à la correction et l'enrichissement de la langue parlée et écrite » (Corbeil, 2007 : 81). Le mandat de l'Office a connu quelques modifications au fil du temps, notamment à la suite des différentes lois linguistiques promulguées en 1969 (loi 63), 1974 (loi 22) et 1977 (loi 101). À l'heure actuelle, l'OQLF, dont le rôle est défini par la Charte de la langue française, a pour mission:

- De définir et de conduire la politique québécoise en matière d’officialisation linguistique, de terminologie ainsi que de francisation de l'Administration et des entreprises.

- De veiller à ce que le français soit la langue habituelle et normale du travail, des communications, du commerce et des affaires dans l'Administration et les entreprises.

- D’aider à définir et à élaborer les programmes de francisation prévus par la loi et en suivre l'application.

- De surveiller l'évolution de la situation linguistique au Québec et d'en faire rapport tous les cinq ans au ministre.

- D'assurer le respect de la Charte de la langue française, agissant d'office ou à la suite de la réception de plaintes.

- D'établir les programmes de recherche nécessaires à l'application de la loi et d'effectuer ou de faire effectuer les études prévues par ces programmes. (OQLF, 2018 : en ligne) 
Autrement dit, en plus de mettre en œuvre des mesures servant à assurer le maintien du statut de la langue française au Québec, l'OQLF recommande une certaine ligne de conduite en matière de langue, visant ainsi à orienter les usages des locuteurs. C'est dans cette perspective d'aménagement de corpus que sont élaborées les politiques de traitement des emprunts linguistiques. C'est aussi dans cette optique que s'inscrit la volonté de franciser une partie de ces emprunts, tout particulièrement des emprunts à l'anglais, notamment par la mise en place d'outils terminologiques (par exemple le Grand dictionnaire terminologique) où sont consignés ces efforts en matière d'aménagement de corpus.

La nouvelle politique de l'emprunt linguistique constitue une version actualisée des politiques précédentes, dont la dernière remontait à 2007. Dans cette toute nouvelle version, l'OQLF, mû par le désir que « ses objectifs en matière de traitement des emprunts soient le plus possible au diapason de [l']évolution [de la langue et de dynamique sociolinguistique] » (OQLF, 2017 : 2), se fixe un objectif triple:

1. Actualiser ses principes et ses critères d'analyse et de traitement des emprunts afin d'en assurer l'adaptation à la situation sociolinguistique québécoise actuelle.

2. Encourager la créativité lexicale en français, qui doit sous-tendre l'évolution du lexique dans toutes les sphères de l'activité humaine, contribuant ainsi à soutenir la vitalité de la langue française.

3. Reconnaître le phénomène de l'emprunt comme un procédé d'enrichissement linguistique productif, dans la mesure où il permet d'intégrer efficacement des formes nouvelles. (OQLF, 2017:4)

La stratégie de l'OQLF « invite à réfléchir de manière rationnelle » (OQLF, 2017 : 3) à l'égard des emprunts. Précisant que son rôle demeure de promouvoir l'usage du français au Québec, l'OQLF opte pour une approche dite nuancée : il écarte les prises de position exclusivement négatives à l'égard des emprunts, estimant que ces derniers peuvent constituer des éléments d'enrichissement de la langue s'ils sont reçus dans la norme de référence du français au Québec et qu'ils s'intègrent au système linguistique du français, tout en rejetant également les prises de position exagérément favorables aux emprunts, qui risqueraient d'entraver la créativité lexicale en français'. Pour y arriver, l'OQLF se fonde sur différents critères pour légitimer ou non certains emprunts linguistiques, et tout particulièrement les emprunts lexicaux : des critères sociolinguistiques (la généralisation, l'implantation et la légitimation de l'emprunt dans l'usage québécois, critères qui tiennent compte notamment de l'aire de distribution géographique et sociale de l'emprunt, de son statut temporel et de sa connotation culturelle), d'une part, et des critères linguistiques (le type d'emprunt, son potentiel d'intégration dans le système linguistique du français et sa coexistence avec d'autres mots et expressions françaises), de l'autre.

1. Il est notamment indiqué dans la politique que « même si un emprunt est accepté par l'Office conformément aux critères énoncés dans la section qui suit, l'organisme, en vertu de sa mission de francisation, favorise néanmoins l'utilisation de mots, de termes et d'expressions français dans le plus grand nombre de cas » (OQLF, 2017 : 8). 
S'il est mentionné dans le document produit par l'OQLF que la nouvelle politique « comporte des principes et des critères de traitement des emprunts qui doivent être suivis dans tous les travaux terminolinguistiques réalisés par l'organisme » (OQLF, 2017 : 4 ; nous soulignons), il n'en demeure pas moins que ces principes sont susceptibles de sortir de ses murs et, donc, d'avoir des répercussions dans l'ensemble de la population québécoise. C'est ce qui s'est produit dès lors que la politique a été diffusée dans les médias, qui se sont immédiatement saisi de la nouvelle. Quelques emprunts sont alors devenus emblématiques sous la plume des journalistes: désormais, ont-ils écrit, il sera légitime pour les Québécois d'employer grilled-cheese, bar à salade et poudre à pâte². Mais entre ces mots porte-étendards de la nouvelle politique de l'emprunt et les véritables motifs et critères derrière les décisions prises par l'Office de même que leurs répercussions sur ce qui peut être qualifié de norme du français québécois, il y a une marge qui n’a pas toujours été exposée de façon claire dans les médias, ce qui a participé à la multiplication des points de vue et à leur confrontation.

\section{Cadre théorique et méthodologique}

\subsection{La nouvelle politique de l'emprunt linguistique : point de départ d'une polémique}

Ces différents points de vue en confrontation constituent le point de départ d'une véritable polémique, soit « un débat autour d'une question d'actualité, d'intérêt public, qui comporte des enjeux de société plus ou moins importants dans une culture donnée » (Amossy, 2014 : 51). Or, plus qu'un simple débat d'idées, la polémique, pour Amossy (2014), constitue une modalité argumentative qui conduit à la dichotomisation des idées jusqu'à les rendre irréconciliables et à la polarisation des groupes qui les défendent, rendant dès lors difficile - voire impossible - l'accord entre les participants au débat et pouvant conduire, à l'extrémité du spectre, à la disqualification de l'adversaire, que ce soit au moyen d'arguments ad hominem (Declercq, 2003) ou de qualifications péjoratives (Laforest et Vincent, 2004). Qui plus est, la polémique se caractérise souvent par un important élargissement du débat (Bernard Barbeau, 2015a) : d'une opposition circonscrite à un événement donné, on en vient à un éclatement des thèmes abordés et à une multiplication des lieux d'affrontement, contribuant dès lors à l'apparence émotive, voire passionnelle, des échanges (Kerbrat-Orecchioni, 1980).

2. Évidemment, la politique de l'emprunt linguistique n'a pas pour fonction d'accorder ou non le droit aux Québécois d'employer certaines formes linguistiques, mais le fait que l'argument selon lequel « les Québécois pourront maintenant dire $X$ » revienne si souvent dans le débat est révélateur de l'image que se font les individus de la mission de l'OQLF et de son rôle dans la société québécoise. Soulignons à titre d'exemple quelques titres d'articles de journaux consacrés à la nouvelle politique de l'emprunt : "Le Québec autorise officiellement ses habitants à dire "cocktail", "legging" et "baby-boom" » (Slate.fr, 23 septembre 2017), " "Le grilled-cheese" and other select anglicisms get stamp of approval from Quebec language watchdog » (National Post, 18 septembre 2017) et «Excuse my French : Quebec language police decides to allow dozens of previously BANNED English phrases into public use » (Daily Mail, 20 septembre 2017). Nous reviendrons sur cet aspect à la section 4.2.1. 
Suivant ces considérations, notre objectif est de faire état de la polémique suscitée par l'annonce dans les médias de l'adoption de la nouvelle politique de l'emprunt linguistique de l'OQLF. Nous montrerons l'opposition qui existe entre les prises de position constitutives du débat et nous nous pencherons sur le glissement qui s'est opéré et qui a fait passer le débat sur la politique de l'emprunt à une discussion plus large sur le rôle de l'OQLF, voire à un conflit révélateur des tensions plus profondément ancrées qui caractérisent le débat linguistique québécois, lui-même traversé d'une multitude d'épisodes polémiques (Bernard Barbeau, 2017, 2018 ; Bernard Barbeau et Molinari, à paraître).

\subsection{Corpus d'analyse}

À cette fin, nous avons constitué un corpus médiatique composé, pour reprendre la typologie de Turbide, Vincent et Kavanagh (2013), de documents journalistiques dits traditionnels (articles, chroniques, éditoriaux, entretiens audiovisuels) et de documents médiatiques citoyens, en l'occurrence des courriers des lecteurs et des billets de blogues.

À partir de la base de données Eureka.cc et du moteur de recherche Google, et des mots clés OQLF+emprunt et OQLF+anglicisme de même que leurs équivalents en anglais, nous avons relevé 39 textes provenant de médias traditionnels abordant la nouvelle politique de l'emprunt. Ces textes ont été publiés entre le 7 septembre et le 28 décembre 2017, majoritairement dans les médias québécois francophones (16 textes), mais aussi anglo-québécois ( 5 textes), canadiens hors Québec (12 textes, tous dans des médias de langue anglaise) et hors Canada (6 textes dans des médias de langue française et anglaise, publiés respectivement en France, en Angleterre, en Écosse et aux États-Unis ${ }^{3}$ ). Nous avons également recueilli 27 billets de blogues, 25 en français et deux en anglais, publiés entre le 26 juillet et le 10 octobre 2017, ainsi que quatre documents audiovisuels (deux entretiens et deux chroniques, tous en français) diffusés entre le 18 septembre et le 19 octobre 2017.

Si l'étendue du corpus montre la place qu'ont occupée les débats dans l'espace public, certains documents sont principalement factuels - c'est le cas des articles qui contiennent uniquement une description non axiologique de la nouvelle politique qui sert à rendre compte de son adoption, et non à juger de son bien-fondé ou non - et ne permettent pas (ou peu) l'analyse de la polémique. C'est pourquoi nous avons plutôt concentré notre attention sur les passages énonciatifs révélateurs d'opinion, c'est-à-dire dans lesquels des jugements à l'endroit de la politique sont étayés à l'aide d'arguments en sa faveur ou en sa défaveur, que ce soit en discours direct (par exemple, dans une lettre d'opinion d'un citoyen ou dans un texte de chroniqueur) ou en discours rapporté (celui, par exemple, d'experts interrogés par des journalistes qui se prononcent sur la politique). Autrement dit, nous avons laissé de côté les éléments de pure description parce que ce ne sont pas eux qui nourrissent la polémique et qui permettent d'observer la confrontation des points de vue.

3. Nous avons également relevé deux articles dans des médias italiens ainsi qu'un article dans un média canado-hongrois. Nous nous sommes cependant concentrées sur les textes en français et en anglais pour notre analyse. 


\section{Analyse}

\subsection{La nouvelle politique de l'emprunt linguistique : quel regard et par qui ?}

Les documents qui constituent notre corpus sont pour la plupart polyphoniques, c'est-à-dire qu'ils présentent plusieurs instances énonciatives (Carel et Ducrot, 2009) auxquelles sont attribués différents points de vue concernant la nouvelle politique de l'emprunt de l'OQLF. Les propos cités dans un document médiatique, par exemple dans un article de journal, sont souvent repris ailleurs, avec pour conséquence que quelques voix, notamment celle de certains linguistes qui interviennent dans le débat, se donnent particulièrement souvent à entendre ou à lire. Compte tenu de cette importante circulation des discours et afin de brosser un portrait plus juste des propos tenus et d'éviter la surreprésentation de certains d'entre eux, nous nous sommes concentrées dans un premier temps sur les différentes instances énonciatives présentes dans le corpus, c'est-à-dire sur les 43 personnes différentes dont les discours, porteurs d'opinion, circulent dans les médias et participent - en raison de leur confrontation - à la polémique.

Nous avons isolé les propos de l'organisme à la source de la polémique, l'OQLF, dont le point de vue est présent dans le corpus à travers la bouche et la plume de ses représentants - employés mais aussi spécialistes qui ont participé aux travaux ayant mené à l'adoption de la nouvelle politique de l'emprunt - de ceux qui relèvent de la réception journalistique, citoyenne et universitaire, où est commentée la politique. Nous avons divisé ces discours de réception entre la parole dite experte ${ }^{4}$ - celle de linguistes ou de chercheurs s'intéressant à la question de la langue, certains ayant d'ailleurs déjà travaillé à l'OQLF mais n'ayant plus de lien d'attache avec l'organisme au moment de l'élaboration de la nouvelle politique de l'emprunt - et non experte, qui inclut autant les citoyens que les journalistes (voir le tableau 1).

Tableau 1. Prise de position des différents types d'instances énonciatives

\begin{tabular}{|c|c|c|}
\hline Instance énonciative & Pour la nouvelle politique & Contre la nouvelle politique \\
\hline OQLF & 4 & 0 \\
\hline Expert & 4 & 5 \\
\hline Non-expert & 23 & 7 \\
\hline
\end{tabular}

Si l'on s'en tient à ces chiffres pris dans leur globalité, on remarque que la nouvelle politique de l'emprunt a été envisagée de façon nettement favorable. Or, apportons quelques précisions en tenant compte de différents paramètres.

4. Sur la distinction entre experts et non-experts, qui a fait l'objet de nombreuses discussions dans le cadre de la linguistique populaire, nous renvoyons notamment à Paveau (2008) et à Visser (2018). 
C'est évidemment sans surprise que les porte-parole de l'OQLF et ceux qui ont participé à l'élaboration de la politique en proposent une vision positive. Il est aussi somme toute peu étonnant que seuls quatre représentants de l'Office se soient prononcés publiquement: leurs fonctions - respectivement directrice générale des services linguistiques, directeur général, porte-parole et présidente du comité d'officialisation linguistique - font en sorte que ce sont eux qui sont appelés à intervenir dans les médias (ou qui, dans le cas de la présidente du comité d'officialisation linguistique, choisissent de le faire par le biais de lettres ouvertes), contrairement à la majorité des autres employés de l'organisme. Mais ce qui peut surprendre, c'est l'espace somme toute restreint qui leur a été accordé dans les médias. Évidemment, les passages descriptifs qui se trouvent, par exemple, dans les articles journalistiques reprennent en large part la politique de l'emprunt, mais on observe malgré tout peu d'entretiens avec les représentants de l'OQLF. Ainsi, le corpus à l'étude comporte un nombre limité de passages expliquant réellement les tenants et aboutissants de la politique de l'emprunt par l'organisme qui en est à l'initiative, ce qui a certainement participé, comme il en sera question plus loin, à un glissement dans le débat.

À l'inverse, une grande importance est accordée au discours expert et non expert. Le fait que neuf experts différents se prononcent dans l'espace public, que ce soit sous forme de discours rapporté dans des articles rédigés par autrui ou encore de façon directe au moyen de lettres d'opinion ou dans des blogues, est suffisamment rare, dans le domaine de la linguistique ${ }^{5}$, pour mériter d'être souligné. Il est également intéressant de noter la division des points de vue : quatre des experts interrogés posent un regard positif sur la nouvelle politique et cinq, un regard négatif. Enfin, les non-experts, qui constituent une forte majorité des instances énonciatives du corpus, envisagent de façon très majoritairement positive la nouvelle politique.

Par ailleurs, on observe un écart majeur entre les différents médias à l'étude. Pour ce qui est des différentes instances énonciatives, c'est dans les médias québécois francophones que la parole experte est principalement rapportée. À l'inverse, ce sont les médias à l'extérieur du Québec francophone qui font entendre de façon majoritaire la voix dite non experte. De plus, ces médias, peu importe leur nature, présentent un regard presque exclusivement positif sur la nouvelle politique de l'OQLF : chroniqueurs, blogueurs, citoyens donnent principalement leur avis sur ce qu'ils considèrent être une ouverture longuement attendue de l'OQLF. Les points de vue sont nettement plus partagés dans les sources québécoises francophones, et il est intéressant de noter que si les journaux traditionnels donnent surtout à voir des opinions défavorables envers la nouvelle politique, c'est dans les entretiens audiovisuels et dans les blogues qu'il est possible de relever le plus grand nombre d'avis positifs.

5. Laforest (1997), comme d'autres d'ailleurs, déplore que la parole des linguistes soit peu prise en considération lorsqu'il est question de langue dans l'espace public. 
En somme, le débat met en scène des intervenants très divers, tant par leur statut (expert ou non) et leur proximité avec l'OQLF que par leur appartenance linguistique, leur provenance géographique et le lieu de diffusion de leurs propos. Les points de vue exprimés, bien que majoritairement orientés en faveur de la nouvelle politique de l'emprunt, laissent entrevoir une importante dissension entre les participants au débat, tout particulièrement entre les experts, très divisés sur la question. Voyons maintenant sur quelle base s'articulent ces différents points de vue.

\subsection{Confrontation des points de vue et élargissement du débat}

\subsection{1. À l'origine de la polémique : la nouvelle politique de l'emprunt de l'OQLF}

En ce qui a trait au point de départ de la polémique, soit la nouvelle politique de l'emprunt même, l'opposition véritablement argumentée entre les points de vue positifs et négatifs s'observe principalement dans le discours des experts, qui ont recours à leur expérience de chercheurs et d'analystes pour poser un regard tantôt approbateur, tantôt critique sur le document présenté par l'OQLF, et, évidemment, dans celui des représentants de l'organisme, qui défendent leur politique.

La première critique énoncée à l'endroit de la politique, qui revient dans les propos de nombreux experts, est que cette dernière a été adoptée en secret, sans que la population en soit informée - ou peut-être davantage sans que les spécialistes du domaine à l'extérieur de l'OQLF aient été consultés avant que soit mise en place cette politique considérée comme le signe d'une nouvelle ère à l'Office. Cette impression est exacerbée par le délai qui s'est écoulé entre l'adoption de la nouvelle politique au sein de l'OQLF, en janvier 2017, et sa diffusion dans l'espace médiatique, en septembre.

(1) Cette révision, qui pourrait être symptomatique de nos rapports de plus en plus décomplexés face à l'anglais, s'est faite sans tambour ni trompette, car je n'ai rien lu ou vu à ce sujet dans les médias. (Jacques Maurais, Le Droit, 7 septembre 2017)

(2) ce que je demande c'est que premièrement cette politique elle a été adoptée en catimini sans qu'on soit véritablement prévenu (Marie-Éva de Villers, 24/60, 18 septembre 2017)

En réponse à ces critiques, c'est sans surprise la voix des représentants de l'OQLF qui se fait entendre. Ces derniers mettent l'accent sur le processus rigoureux et transparent qui a mené à l'adoption de la nouvelle politique de l'emprunt. La longueur du travail mené par le Comité d'officialisation linguistique, le nombre de spécialistes ayant participé au processus décisionnel et le demi-siècle d'existence de l'OQLF jouent alors le rôle d'arguments d'autorité qui témoignent du professionnalisme avec lequel l'organisme a mené ce dossier et qui garantissent le bien-fondé de la nouvelle politique: 
(3) L’Office, qui a 56 ans, peut-il tenir compte de l'évolution du Québec et adopter une stratégie d’emprunt linguistique aussi réfléchie que les précédentes? Assurément. Et il applique tout le sérieux et toute la rigueur que commande l'exercice. Le Comité d'officialisation linguistique que j'ai présidé a pu analyser et commenter l'état d'avancement du travail - qui s'est échelonné sur trois ans - des nombreux terminologues affectés à ce dossier. C'est entièrement satisfait de la version aboutie de la politique que le Comité lui a donné son aval. (Monique Cormier, Le Devoir, 27 septembre 2017)

La rigueur de ce processus est toutefois remise en question - parfois de façon très vive - par les détracteurs de la politique. Leur regard négatif est justifié par ce qui est considéré comme d'importants manquements méthodologiques et des irrégularités dans l'élaboration même de la politique. Ces aspects sont très nombreux, et la plupart des experts qui se prononcent en défaveur de la politique y font référence, que ce soit de façon générale (exemple 4), à partir d'exemples précis qui apparaissent incohérents au regard des critères adoptés par l'OQLF (exemple 5) ou encore en référence au postulat même qui a guidé les travaux de l'organisme (exemple 6). Ces critiques sont également reprises dans le discours de certains non-experts, comme c'est le cas dans l'exemple 7, où un éditorialiste commente à son tour ce qu'il présente comme des lacunes scientifiques et un flou dans l'élaboration de la politique.

(4) L'absence de fondements scientifiques vérifiables se cache derrière l'emploi de multiples critères d'acceptation ou de rejet des emprunts aussi hétéroclites que peu crédibles. (Lionel Meney, blogue, 10 octobre 2017)

(5) Mon propos n'est pas de discuter de la légitimité des anglicismes en français. Je veux plutôt attirer votre attention sur les maladresses, voire les erreurs, d'un document produit à même les fonds publics en vous posant quatre questions très simples : Comment peut-on affirmer que le mot selfie (p. 14), utilisé quotidiennement par des millions de francophones, ne s'intègre pas au système linguistique du français? Vous prenez selfie, vous changez deux lettres et vous avez Sylvie : selfie n'est pas plus difficile à écrire et à prononcer que Sylvie. [...] (Jacques Maurais, Le Soleil, 4 décembre 2017)

(6) Dans le préambule de sa nouvelle politique, l'OQLF écrit: "La langue et la dynamique sociolinguistique étant en évolution constante, l'Office se doit de mettre sa politique à jour régulièrement afin que ses objectifs en matière de traitement des emprunts soient le plus possible au diapason de cette évolution ». La situation linguistique québécoise a-t-elle progressé au point qu'il soit maintenant justifié d'assouplir les critères d'acceptation des anglicismes? Poser la question, c'est y répondre. (Jean-Claude Corbeil et Marie-Éva de Villers, Le Soleil, 24 septembre 2017) 
(7) Voulant adoucir son image d'ayatollah de la langue, l'OQLF ouvre la porte, avec sa nouvelle politique, à l'acceptation d’anglicismes dans la mesure où ils sont "non récents", "généralisés" et "légitimés", des critères pour le moins discutables, voire nébuleux. (Robert Dutrisac, Le Devoir, 21 septembre 2017)

Du côté des défenseurs de la politique, à l'inverse, la logique derrière les choix de l'OQLF est évoquée, principalement parce que la nouvelle politique de l'emprunt est réputée plus cohérente avec les pratiques linguistiques réelles des Québécois. S'ensuit alors une discussion sur la question de l'usage - les métaphores guerrières sur la force de l'usage et sur le fait que ce dernier l'emporte malgré les tentatives qui sont faites pour le réguler sont abondantes - et à propos de recommandations antérieures de l'OQLF qui n'ont jamais été adoptées par les locuteurs (par exemple gaminet ou encore la graphie coquetel), données comme preuve de l'importance d'une plus grande souplesse de la part de l'organisme ou, à tout le moins, d'une plus grande prise en compte des pratiques des locuteurs.

(8) Melançon gave the provincial language agency top marks for having a "realistic" policy which also encourages the use of French words. "It's not worth going to war over "grilledcheese" because it's common usage”, he added. (Benoît Melançon, cité par Peter Rakobowchuk, Metro News, 19 septembre 2017)

(9) Les autorités langagières ont pas juridiction sur la langue quotidienne [...] c'est toujours l'usage qui gagne (Anne-Marie Beaudoin-Bégin, Québec, réveille!, 27 septembre 2017).

On trouve dans ces extraits la remise en discours des propositions de l'OQLF, dont la nouvelle politique se veut justement plus en phase avec les pratiques linguistiques des Québécois. L'argument de l'adéquation avec l'usage réel, que l'on observe dans les exemples précédents, est donc doublement présent dans le corpus: dans le discours d'experts en faveur de la politique et dans celui de représentants de l'OQLF, qui s'en servent pour légitimer leurs propositions. Certains d'entre eux ajoutent que l'emprunt, phénomène observable dans toutes les langues, peut constituer une forme d'enrichissement linguistique :

(10) en fait l'Office québécois de la langue française continue d'encourager le renforcement des compétences linguistiques de l'ensemble des locuteurs québécois et ceux de la francophonie en général, l'Office continue de préconiser et d'encourager, de stimuler la créativité lexicale en langue française, et du même souffle, c'est vrai que nous admettons ce qui est déjà admis par la plupart des spécialistes, que l'emprunt linguistique peut être une façon normale d'enrichir la langue (Robert Vézina, TV5 monde, 19 octobre 2017) 
Cette volonté de se rapprocher de l'usage est toutefois fortement critiquée puisqu'elle est jugée contraire au mandat confié à l'OQLF par l'article 159 de la Charte de la langue française, qui est d'orienter les usages et d'établir (voire de prescrire) une certaine norme linguistique. En officialisant sa nouvelle politique de l'emprunt, l'organisme est alors perçu comme ayant changé de rôle pour adopter une approche descriptive habituellement réservée aux linguistes, et non aux instances chargées d'établir des recommandations en matière de langue :

(11) Je me demande si l'Office, qui n'a pas officiellement changé de mandat, n'aurait pas changé de mandat officieusement [...]. Son rôle, ce n'est pas de décrire l'usage et de déterminer ce qui est légitime et ce qui ne l'est pas, c'est d'orienter l'usage. J'ai l'impression que l'OQLF est en train de transformer son rôle de représentant en observateur de la norme. J'ai l'impression qu'il y a des linguistes à l'office qui ont envie d'être linguistes en décrivant l'usage, pour dégager une norme. Non. Le rôle de l'Office, ce n'est pas de décrire l'usage, mais de l'orienter. (Nadine Vincent, Le Devoir, 18 septembre 2017)

(12) La mission de l'organisme est d'assurer la définition et la diffusion par le GDT des terminologies françaises des différents domaines d'emploi. À cet égard, son rôle ne s'exerce pas dans tous les registres de langue : il se limite aux registres de la langue technique courante ou soutenue. II n'entre pas dans les attributions de l'OQLF de décrire et de légitimer les emplois de registre familier. (Jean-Claude Corbeil et Marie-Éva de Villers, Le Soleil, 24 septembre 2017)

Or, ce changement de cap suscite tout particulièrement l'indignation chez les experts, dont quatre des cinq qui se prononcent en défaveur de la politique ont recours à cet argument pour justifier leur prise de position. Affirmant que l'OQLF sert de référence vers laquelle se tourne la population lorsqu'il s'agit de connaître l'acceptabilité de certains termes, ils s'inquiètent de l'effet de cette nouvelle mission que semble s'être confiée l'organisme. Le rôle de l'Office est donc au cœur de la discussion et soulève des questionnements qui vont bien au-delà de la question des emprunts et qui doivent faire l'objet d'une réflexion collective approfondie:

(13) Alors, que nous utilisions tous le mot grilled cheese parce que sandwich au fromage fondant n'a pas passé la rampe de l'usage n'a aucune importance dans le débat actuel. Ce qu'il faudrait déterminer collectivement, c'est si l'Office québécois de la langue française peut changer son mandat et ne faire que décrire l'usage ou s'il doit continuer à l'orienter ; 'il doit se contenter de servir de miroir à la langue en usage au Québec ou s'il doit persévérer à lui proposer des fenêtres sur de nouvelles perspectives. (Nadine Vincent, Le Devoir, 29 septembre 2017) 
En somme, le débat montre comment les experts - des spécialistes de la description linguistique - sont divisés sur la question de la nouvelle politique de l'emprunt. Or, on constate que l'opposition est plus complexe qu'une « simple » guerre idéologique qui opposerait des spécialistes de la langue selon des camps bien définis, par exemple les tenants de la prescription contre ceux de la description ou encore les défenseurs d'une norme endogène contre ceux d'une norme exogène, typique du débat entre linguistes québécois dans les dernières décennies (Poirier, 1998 ; Pöll, 2009). Bien au contraire, tantôt la division s'opère entre des chercheurs qui partagent pourtant par ailleurs de nombreux points de vue sur la langue (approche descriptive et pluricentrique de la langue, en faveur de l'établissement d'une norme endogène du français québécois, etc.), tantôt le débat réunit des chercheurs qui ne logent pourtant pas à la même enseigne quand vient le temps de se prononcer sur la variété québécoise de français (ceux qui prônent un français dit « international » et qui voient en l'établissement d'une norme endogène au Québec une forme de ghettoïsation, et ceux qui, au contraire, œuvrent à la description et à la valorisation du français en usage au Québec). Cette polarisation du débat, typique de la polémique, témoigne du véritable questionnement des experts à propos du bien-fondé de la nouvelle politique de l'emprunt de l'OQLF, du mandat de l'organisme et des effets possibles d'un changement de cap de sa part.

Chez les non-experts, le regard posé sur la politique même est plus rare et il est également moins argumenté. Lorsqu'il est présent dans les discours analysés, c'est presque exclusivement en référence à des cas précis - mots, syntagmes, expressions - envisagés tantôt avec inquiétude parce qu'ils sont vus comme un indice que la « lutte à l'anglicisation » a échoué (exemple 14), tantôt avec bonheur et jubilation, voire avec un sentiment de liberté linguistique nouvellement acquise (exemple 15, où l'auteur se penche sur le mot grilled-cheese):

(14) Faudrait-il revenir en arrière et accepter en français des anglicismes que nous avons presque réussi à éradiquer comme bumper, wipers, flat, etc. (Pierre Lincourt, Le Soleil, 20 septembre 2017)

(15) In fact, I'm getting gooey about the prospect of additional culinary items being added to menus across the province as I realize the potential impact of a sudden legal liberation from linguistic constraint and freedom. (Greg Duncan, Montreal Gazette, 25 septembre 2017)

Il est aussi question - et ce probablement en raison d'une incompréhension ou, du moins, d'une compréhension incomplète de la politique - de ce qui est présenté comme un droit nouvellement acquis pour les Québécois, soit celui d'employer certaines formes linguistiques. On observe cet élément principalement dans les médias anglophones et hors Québec : 
(16) English-language words such as hashtag, grilled-cheese, and parking are now acceptable in everyday French-language conversation in Quebec society, according to guidelines recently updated by the province's language watchdog. (Peter Rakobowchuk, Metro News, 19 septembre 2017)

(17) Selon les nouvelles règles, le mot « grilled-cheese » pourra aussi être utilisé au lieu de «sandwich au fromage fondant » (Claire Levenson, Slate.fr, 23 septembre 2017).

Or, soulignons que les mots donnés ici en exemple sont déjà bien ancrés dans l'usage de nombre de Québécois ; il ne s'agit donc pas d'une nouveauté découlant de l'intervention de l'OQLF. Par ailleurs, hashtag et parking ne figurent pas dans la nouvelle politique de l'Office, et le terme mot-clic, pour hashtag, est souvent cité en exemple d'option française bien intégrée dans l'usage québécois. Ces exemples, qui circulent abondamment dans les discours journalistiques et citoyens, reposent sur une lecture sinon absente, du moins partielle, de la nouvelle politique. Par ailleurs, et surtout, la question du « droit » d'employer ces mots est à relativiser : nous l'avons mentionné, le mandat de l'OQLF n'est pas de régir l'usage quotidien des locuteurs. Cette perception erronée du rôle de l'Office comme instance de surveillance ayant droit de vie ou de mort sur certains usages contribue à élargir le débat, qui dépasse très rapidement le cadre strict de l'emprunt linguistique.

\subsubsection{Au-delà de la politique de l'emprunt, les tensions linguistiques au Québec}

Dans nombre de controverses linguistiques au Québec, l'élément déclencheur, point de départ aux discussions, sert de prétexte pour revenir sur des tensions plus profondément ancrées dans la société, ce qui confère au débat une certaine profondeur sociohistorique contribuant à en faire une véritable polémique. Il est ainsi fréquent que les échanges, alors particulièrement virulents, se construisent autour d'éléments porteurs sur le plan mémoriel, divisifs et moteurs de ressentiment (Bernard Barbeau, 2015b).

Dans le cas de la polémique qui nous intéresse, il est fréquent que les participants au débat aient recours à la nouvelle politique de l'emprunt pour faire état de considérations politiques, et notamment pour critiquer plus largement le gouvernement et son manque d'intérêt envers la question linguistique :

(18) Il est évident que notre gouvernement ne semble pas très actif dans le domaine de la langue. Quant au ministre responsable de l'application de la charte de la langue française, il est plutôt timide, pour ne pas dire absent, dans sa défense de la langue. (Pierre Lincourt, Le Soleil, 20 septembre 2017) 
Nombreux aussi sont ceux qui profitent de la tribune qui leur est offerte pour aborder la situation sociolinguistique québécoise et pour présenter la population francophone parfois comme menacée par l'anglais, menace qui serait accentuée par la nouvelle politique de l'OQLF (19), parfois comme plus ouverte à la question de l'emprunt en raison d'une plus grande sécurité linguistique (20), voire comme «enfin libérée » - l'expression revient d'ailleurs à quelques reprises, toujours dans le discours de non-experts - de son complexe par rapport à l'anglais et de sa peur à son égard.

(19) Or, l'anglicisation massive du vocabulaire a touché toute la société - à tel point qu'un ancien directeur de l'Office, Jean-Claude Corbeil, a dit que « l'action de l'Office [depuis ses débuts en 1961] a été une entreprise de décolonisation » (L'Actualité, avril 1989). Comprenons que l'on met fin à cette entreprise de décolonisation. [...] À l'Office québécois de la langue française, l'ère de la décolonisation a pris fin. Commence maintenant l'ère de l'asservissement volontaire. (Jacques Maurais, Le Droit, 7 septembre 2017)

(20) Quebecers are now ready for opening up a little bit about the English words. [...] Younger people probably feel more at ease with French and they are more bilingual and they don't seem to perceive English as such a menace or as threatening as they used to. (Chantal Bouchard, CBC Montreal, 19 septembre 2017)

Sensiblement dans la même veine, le rapport de force historiquement inégal entre francophones et anglophones, les luttes linguistiques des francophones dans les années 1960-1970 et leurs effets sur les groupes linguistiques, quatre décennies plus tard, sont fréquemment mis de l'avant par les participants au débat:

(21) Collectively, francophone Quebeckers have improved their status economically and politically. There are still insecurities, but there are fewer than before. (Chantal Bouchard, The Globe and Mail, 18 septembre 2017)

(22) L'énoncé de politique n'offre pas de cadre historique expliquant le grand nombre d'anglicismes dans le français du Québec. Évacuation donc de l'histoire. Ne surtout pas parler de la Conquête et de ses conséquences. Les rédacteurs de l'énoncé ont oublié les leçons de leur maître. (Jacques Maurais, blogue, 3 août 2017)

(23) Not only that, but the OQLF says these changes and others like them are "part of our linguistic enrichment". For English-speaking Quebeckers, it's a bit much. Their mother tongue has been demonized, and even banned from public view, in their home province. To now be told that English words contribute to the "linguistic enrichment" of French is annoying (a feeling best expressed by an English word borrowed from... French). (éditorial, The Globe and Mail, 20 septembre 2017) 
Ces éléments, d'ailleurs remis en circulation dès lors qu'est abordée la question linguistique dans l'espace public, constituent de véritables lieux de mémoire qui traversent les débats sur la langue, peu importe ce qui en est le déclencheur. Toute polémique linguistique en contexte québécois est susceptible de voir l'un ou l'autre de ces rappels historiques faire l'objet d'une thématisation, dont l'orientation - positive ou négative - participe à la dichotomisation des points de vue et, souvent, contribue à la force émotive des discours tenus. Cela est particulièrement manifeste dans les exemples 22 et 23 cités précédemment, tous deux empreints d'une vive rancœur découlant du rapport entre les groupes linguistiques, bien que cette rancœur repose sur des raisons différentes.

L'élargissement de la polémique se manifeste également par de nombreux discours où il est question des actions de l'OQLF au fil du temps, et non seulement en lien avec la politique de l'emprunt, et ce afin de remettre en question la pertinence de l'organisme ou, du moins, pour critiquer ce qui est considéré comme une attitude intransigeante de sa part:

(24) For decades, the guardians of the French language in Quebec have waged a relentless battle against encroachments of English into everyday life. So it's no small matter that the watchdogs have given their blessing to the humble grilled-cheese sandwich. (Ingrid Peritz, The Globe and Mail, 18 septembre 2017)

Les individus ont alors recours à des événements antérieurs qui mettent en scène l'OQLF pour étayer leur argumentation. C'est notamment le cas de ce qui a depuis été qualifié de pastagate, qui revient à plusieurs reprises dans le corpus. Ce faisant, la mission de l'OQLF est présentée comme excessive, voire absurde. Cela se manifeste aussi par l'emploi de certaines formes linguistiques connotées négativement et servant à disqualifier l'organisme. Les appellations language watchdog, language police ou fusty stickler for "pure French", que l'on trouve dans les propos de non-experts dans les médias de langue anglaise, sont révélatrices du regard jeté sur l'organisme et confèrent aux discours qui les contiennent une forte tonalité négative. Cela d'autant plus marqué que le vocabulaire employé pour parler de la nouvelle politique de l'emprunt est quant à lui plutôt positif : mature approach, good news, common sense, flexibility, etc.

D’un débat sur la nouvelle politique de l'emprunt linguistique, la polémique s'étend donc pour laisser la place à une discussion plus générale sur l'OQLF et sur sa pertinence, sur la situation linguistique québécoise et sur le rapport entre les groupes linguistiques et les tensions qui les animent. Si ces propos sont tenus autant par des experts que par des non-experts, la dimension émotive transparaît surtout du discours des derniers, et plus particulièrement de ceux qui sont en faveur de la nouvelle

6. Rappelons pour mémoire que l'affaire a commencé quand un client a porté plainte à l'OQLF, en 2013, après avoir reçu un menu unilingue anglais dans un restaurant italien de Montréal. Or, l'employé de l'OQLF chargé d'analyser la situation a plutôt envoyé un avertissement au propriétaire du restaurant, l'enjoignant de supprimer les mots italiens du menu (antipasti, par exemple) et de les remplacer par des équivalents français. Ce n'est qu'après de virulents échanges dans les médias et de vives critiques de l'OQLF, accusé de zèle, que le motif initial de la plainte a été rappelé : le menu en langue anglaise remis au client, et non la présence de l'italien pour désigner certains plats. Pour une présentation détaillée de la polémique et des idéologies linguistiques qui la traversent, voir Vessey (2016). 
politique, qui sont nombreux à aborder ce qu'ils estiment être des injustices ou des aberrations de la part de l'OQLF, ouvrant ainsi la porte à des disqualifications parfois virulentes de l'organisme.

\section{Conclusion}

Les échanges autour de la nouvelle politique de l'emprunt linguistique de l'OQLF constituent un terreau fertile pour étudier la façon dont s'est construite et articulée la polémique à ce sujet. L'analyse menée a permis de montrer que la polémique se joue sur deux terrains. D'un côté, les experts s'interrogent principalement sur les critères adoptés par l'OQLF dans sa nouvelle politique et sur le mandat confié à l'organisme, ou encore abordent la situation sociolinguistique québécoise dans une perspective historique. De l'autre, les non-experts s'intéressent principalement à ce qui est présenté comme la soudaine libération de la langue au Québec et à l'assouplissement d'un organisme présenté comme contraignant, ce qui participe à l'élargissement du débat, aux relations conflictuelles entre les groupes linguistiques au Québec et au rôle qu'y joue la nouvelle politique de l'emprunt.

Les différents arguments proposés en faveur ou en défaveur de la nouvelle politique de l'OQLF témoignent d'une véritable relation polémique, telle que définie par Amossy (2014), à savoir une forme de coexistence dans le dissensus. Autrement dit, loin d'aboutir à un accord, les participants au débat sont campés dans des positionnements dichotomiques qui se déploient par le biais de dispositifs argumentatifs différents, sans jamais parvenir à une véritable interaction. On voit alors apparaître ce qu'Angenot (2008) qualifie de dialogue de sourds, où le débat sert alors davantage de lieu d'expression individuelle que de véritable terrain d'échange et d'entente. En ce sens, les discussions autour de la nouvelle politique de l'emprunt linguistique de l'OQLF, où l'on observe la circulation d'arguments présents dans d'autres débats et la remise en discours d'événements du passé et de positionnements idéologiques qui en découlent, contribuent à mettre en évidence le fonctionnement des polémiques linguistiques québécoises, mais aussi, plus largement, les points de dissension qui perdurent au fil du temps. 


\section{Références}

Amossy, Ruth (2014), Apologie de la polémique, Paris, Presses universitaires de France.

Angenot, Marc (2008), Dialogues de sourds. Traité de rhétorique antilogique, Paris, Mille et une nuits.

Bernard Barbeau, Geneviève (2018), « 40 ans après, qu'en est-il de la loi 101 ? Représentations et discours conflictuels dans la presse québécoise », Circula : revue d’idéologies linguistiques, n 7 , p. 51-69.

Bernard Barbeau, Geneviève (2017), « Disqualification d'autrui, disqualification de soi : l'auto-dévaluation chez les Québécois francophones », Minorités linguistiques et société, n 8, p. 83-101.

Bernard Barbeau, Geneviève (2015a), Québec bashing. Analyse du discours entourant l'affaire Maclean's, Montréal, Éditions Nota bene.

Bernard Barbeau, Geneviève (2015b), "L'affaire Maclean's entre critique légitime, bashing et violence détournée : le rôle du ressentiment dans l'interprétation d'un discours controversé », Semen, n 40, p. 111-118.

Bernard Barbeau, Geneviève et Chiara Molinari (à paraître), «Bonjour/hi ou quand la polémique arrive par les mots », dans Geneviève Bernard Barbeau, Franz Meier et Sabine Schwarze (dir.), Conflits sur/dans la langue: approches linguistiques, argumentatives et descriptives, Francfort, Peter Lang.

Bouchard, Chantal (1999), On n'emprunte qu'aux riches : la valeur sociolinguistique et symbolique des emprunts, Montréal, Fidès.

Carel, Marion et Oswald Ducrot (2009), « Mise au point sur la polyphonie », Langue française, $n^{\circ}$ 164, p. 33-43.

Corbeil, Jean-Claude (2007), L'embarras des langues. Origine, conception et évolution de la politique linguistique québécoise, Montréal, Québec Amérique.

Declercq, Gilles (2003), "Avatars de l'argument ad hominem : éristique, sophistique, dialectique », dans Gilles Declercq, Michel Murat et Jacqueline Dangel (dir.), La parole polémique, Paris, Champion, p. 327-376.

Kerbrat-Orecchioni, Catherine (1980), Le discours polémique, Lyon, Presses universitaires de Lyon.

Laforest, Marty (1997), États d’âme, états de langue. Essai sur le français parlé au Québec, Québec, Nota bene.

Laforest, Marty et Diane Vincent (2004), «La qualification péjorative dans tous ses états », Langue française, nº 144 , p. 59-81.

Office québécois de la langue française (2018), Mission et rôle de l'organisme, disponible sur https:// www.oqlf.gouv.qc.ca/office/mission.html. [Page consultée le 28 janvier 2019.] 
Office québécois de la langue française (2017), Politique de l'emprunt linguistique, Québec, Office québécois de la langue française.

Paveau, Marie-Anne (2008), « Les non-linguistes font-ils de la linguistique ? Une approche anti-éliminativiste des théories folk », Pratiques, nos 139-140, p. 93-109.

Poirier, Claude (1998), « De la défense à la codification du français québécois : plaidoyer pour une action concertée », Revue québécoise de linguistique, vol. 26, n², p. 129-150.

Pöll, Bernhard (2009), «Internationalisants contre aménagistes : petit essai d'analyse d'une guerre d'idéologies linguistiques », dans Béatrice Bagola (dir.), Français du Canada - français de France. Actes du huitième Congrès international de Trèves, Tübingen, Max Niemeyer Verlag, p. 71-80.

Turbide, Olivier, Diane Vincent et Éric Kavanagh (2013), «Repères méthodologiques pour l'analyse des discours sociaux: Lorsqu'un tweet devient une "affaire" », dans Danielle Londei, Sophie Moirand, Sandrine Reboul-Touré et Licia Reggiani (dir.), Dire l'événement: langage mémoire société, Paris, Presses Sorbonne Nouvelle, p. 23-34.

Vessey, Rachelle (2016), « Language ideologies in social media : the case of Pastagate », Journal of language and politics, vol. 15, n 1, p. 1-24.

Vincent, Nadine (2017), «L'élite du Québec à l'assaut du français québécois : 150 ans de dénigrement dans la presse écrite », dans Paola Puccini et Isabelle Kirouac Massicotte (dir.), Langue et pouvoir, Bologne, Casa Editrice Clueb, p. 53-73.

Visser, Judith (2018), « Linguiste ou non-linguiste ? Réflexions sur une dichotomie controversée à partir de l'analyse de métadiscours sur les langues régionales », Les carnets du Cediscor, n 14 , p. 88-102. 